\title{
CONTROL OF TRANSONIC CAVITY FLOW INSTABILITY BY STREAMWISE AIR INJECTION
}

\author{
Aldo Rona* \\ Department of Engineering, University of Leicester, Leicester, LE1 7RH, UK
}

\begin{abstract}
A time-dependent numerical model of a turbulent Mach 1.5 flow over a rectangular cavity has been developed, to investigate suppression strategies for its natural self-sustained instability. This instability adversely affects the cavity form drag, it produces largeamplitude pressure oscillations in the enclosure and it is a source of far-field acoustic radiation.

To suppress the natural flow instability, the leading edge of the two-dimensional cavity model is fitted with a simulated air jet that discharges in the downstream direction. The jet mass flow rate and nozzle depth are adjusted to attenuate the instability while minimising the control mass flow rate.

The numerical predictions indicate that, at the selected inflow conditions, the configurations with the deepest nozzle ( 0.75 of the cavity depth) give the most attenuation of the modelled instability, which is dominated by the cavity second mode. The jet affects both the unsteady pressure field and the vorticity distribution inside the enclosure, which are, together, key determinants of the cavity leading instability mode amplitude. The unsteadiness of the pressure field is reduced by the lifting of the cavity shear layer at the rear end above the trailing edge. This disrupts the formation of upstream travelling feed-back pressure waves and the generation of far-field noise. The deep nozzle also promotes a downstream bulk flow in the enclosure, running from the upstream vertical wall to the downstream one. This flow attenuates the large-scale clockwise recirculation that is present in the unsuppressed cavity flow. The same flow alters the top shear layer vorticity thickness and probably affects the convective growth of the shear layer cavity second mode.
\end{abstract}

\section{BACKGROUND}

A rectangular recess, or cavity, open to a transonic air stream can experience large amplitude aerodynamic loads, due to unsteady flow developing in and around the enclosure. In aircraft, such loads can develop in an open store bay, subjecting airframe and stores to unwanted structural vibration that could undermine their integrity. ${ }^{1}$ Safe store separation is also a concern, as light stores may impact against the bay walls or against neighbouring stores, when released,

\footnotetext{
* Lecturer.
}

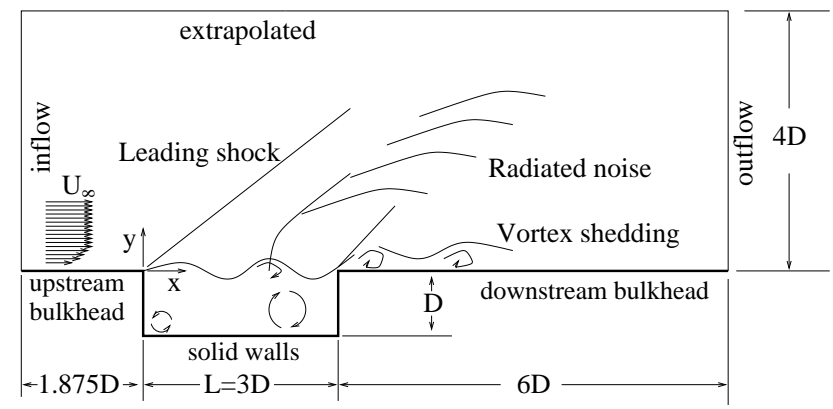

Fig. 1 Sketch of the transonic flow past an open cavity.

due to the unsteady flow. At transonic flow regimes, aircraft store bays have been a very prominent application for cavity flow research in recent years, ${ }^{2}$ as current airframes make extensive use of weapon bays for stealth and store protection during cruise.

The flow unsteadiness in a transonic cavity is driven by a natural flow instability, which is diagrammatically represented in Fig. 1. This develops by the coupling of a convectively amplifying Kelvin-Helmholtz type shear-layer instability across the cavity opening with large-amplitude pressure oscillations, an unsteady vorticity field and flow recirculation in the enclosure. The amplitude of these pressure oscillations can be as large as $150 d B$ re $20 \mu P a$. In the cavity, these give rise to sustained aerodynamic loads and pressure drag. In the far-field, part of this pressure fluctuation is perceived as aerodynamic noise.

There has been a sustained interest to understand and suppress the instability of transonic cavity flows, motivated by the scientific challenge that this problem poses and by the potential rewards from this research to the aeronautical industry. Past experiments ${ }^{3-5}$ and numerical studies ${ }^{2,6}$ have tested a range of suppression strategies for cavity flows. These have included the use of ramps, ${ }^{7-11}$ spoilers, rods ${ }^{12}$ and air jets ${ }^{4,13,14}$ located mainly on the upstream bulkhead, to affect the oncoming boundary layer approaching the enclosure. Some configurations considered ramps located at the downstream bulkhead, to alter the reattachment of the boundary layer. ${ }^{7}$ Investigations have also been conducted with active control devices, such as vibrating ramps $^{1,15}$ or synthetic ${ }^{4}$ or pulsating jets. ${ }^{4,14,16}$ The design of an effective feedback controller for such active suppression devices still poses a great challenge to the

1 of 10

American Institute of Aeronautics and Astronautics 


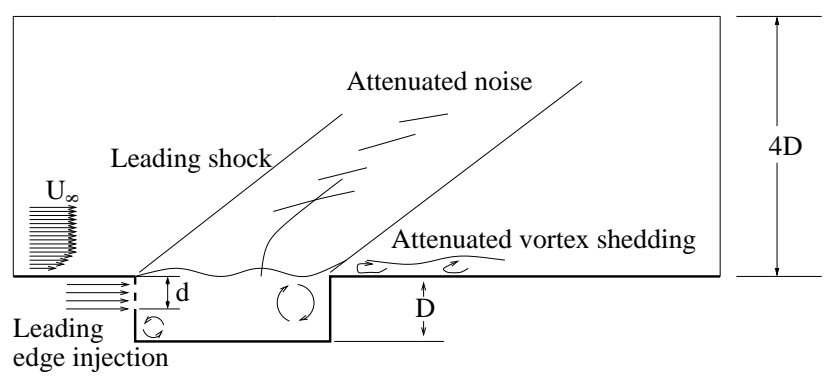

Fig. 2 Sketch of the transonic cavity flow with mass injection.

scientific community and has led to a number of scientific contributions to the control of cavity flows. ${ }^{17-20}$

This paper aims to further explore configurations of instability attenuators, applicable to transonic turbulent cavity flows. Rona \& Brooksbank ${ }^{14}$ investigated by numerical modelling the use of air jet mass injection as attenuators at different locations around the enclosure perimeter. This investigation concluded that injecting air from the upstream cavity bulkhead in the downstream direction was the most promising configuration among the ones tested by Rona \& Brooksbank. ${ }^{14}$ This best performing configuration is diagrammatically shown in Fig. 2. In the current work, the injection depth $d$ (Fig. 2) is adjusted to further improve the control on the flow.

A Mach 1.5 air flow over a rectangular enclosure of length to depth ratio 3 , diagrammatically shown in Fig. 1, is the benchmark test case to assess the performance of different flow suppression configurations. This test case is documented by several contributions to the literature. ${ }^{8-11,14,19,20}$ At this regime, the cavity flow is 'open' 21 and its instability is driven by the fluctuations of a shear layer that spans across the enclosure. ${ }^{21}$ At the selected inflow conditions, the interaction of the shear layer with the rear bulkhead sets up a feed-back loop that self-sustains the instability. The cavity flow dynamics is dominated by the motion of large scale flow structures ${ }^{22-24}$ and features strong indications of a limit-cycle instability. These structures are the energy containing eddies in the shear layer and are the main drivers of the self-sustained flow unsteadiness. Past numerical work ${ }^{23-26}$ has shown that these structures can be modelled successfully by an appropriate time-dependent two-dimensional numerical method. This method resolves directly in space and time the large-scale motion in the flow and uses a turbulence model to account for the effects of the unresolved scales of motion on the macro-dynamics of the flow. This approach is continued in the present study. A broader account of numerical, analytical and experimental approaches for cavity flows is given in a review by Grace. ${ }^{6}$

\section{INFLOW CONDITIONS}

Figure 1 shows the computational domain and the geometry of the rectangular cavity modelled in this study. The length to depth 3 enclosure and transonic inflow are designed to match the experimental conditions of a selected 'open' turbulent cavity test by Zhang. ${ }^{25}$ A turbulent boundary layer develops above the upstream cavity edge. All dimensions are normalised by the $15 \mathrm{~mm}$ cavity depth $D$. At the computational domain inflow boundary, the boundary layer thickness $\delta_{99}=0.333 D$. Above the boundary layer, the uniform free stream air speed $U_{\infty}=425.2 \mathrm{~m} / \mathrm{s}$, the Mach number $M_{\infty}=1.5$, the density $\rho_{\infty}=$ $0.9373 \mathrm{~kg} / \mathrm{m}^{3}$, the static pressure $p_{\infty}=53.801 \mathrm{kN} / \mathrm{m}^{2}$ and the static temperature $T_{\infty}=200 \mathrm{~K}$. Injection is performed through the upstream bulkhead, as shown in Fig. 2. The injection depth varies in the range $0 \leq d \leq 0.75 D$.

\section{NUMERICAL METHOD}

A two-dimensional finite-volume second-order accurate time-marching scheme from Rona \& Brooksbank $^{14}$ provides time-dependent predictions of the unsteady cavity flow, with and without mass injection. This numerical method is an extension of a time-dependent numerical scheme for laminar flows by Rona \& Dieudonné. ${ }^{27}$ It uses an approximate Riemann solver and min-mod flux limiter by $\operatorname{Roe}^{28,29}$ to estimate the convective fluxes at the finite-volume unit-cell boundaries and it uses second order central differences for the diffusive fluxes. A two-step RungeKutta algorithm with standard coefficient 0.5 and 1.0 integrates the compressible flow governing equations explicitly in time. In the extended finite volume method, the model flow governing equations are the discrete time-dependent Reynolds Averaged NavierStokes equations with $k-\omega$ turbulence closure and $\omega$ cross-diffusion. ${ }^{30}$ The computational domain boundaries are located as shown in Fig. 1. A body-fitted structured uniform rectangular multi-block mesh is used to define the finite volumes or unit cells for the application of the conservative laws. A $40 \times 40$ mesh is used inside the enclosure and a $145 \times 200$ mesh is used above the opening. This gives a constant unit cell aspect ratio $\Delta x / \Delta y=3$. The numerical model is further detailed in Rona \& Bennett ${ }^{31}$ and in Rona \& Brooksbank. ${ }^{14}$

Injection is considered through the upstream bulkhead in the downstream direction. For this configuration, a perforated cavity wall is used. Injection is performed through a surface of constant void-ratio and a constant back pressure $p_{0}$ is imposed below the surface. The injection depth $d$ varies between 0 (no injection) and $0.75 D$, below the cavity edge. The wall normal velocity $v_{w}$ is estimated from the linear form

2 of 10

American Institute of Aeronautics and Astronautics 
of the Darcy pressure-velocity law, ${ }^{14}$ from which

$$
v_{w}=\frac{\sigma}{\rho_{\infty} U_{\infty}}\left(p_{0}-p_{w}\right)
$$

where $\sigma=0.607$ is the geometric porosity. ${ }^{32} p_{w}$ is the static pressure extrapolated from the internal domain conditions adjacent to the wall.

To compare the result from tests with different injection depths and injection back pressures, two parameters are defined that qualify the cost of providing flow control and the benefits obtained from any attenuation of the flow instability. Consistently with previous work, ${ }^{14}$ these parameters are the mass flow rate supplied to the perforated wall $\left\langle\rho v_{w}\right\rangle$ and the cavity pressure drag coefficient $C_{d}$. The space averaged mass flow rate through the perforations is estimated from

$$
<\rho v_{w}>=\frac{1}{\rho_{\infty} U_{\infty} d} \int_{-d}^{0} \rho v_{w} d y
$$

and $\left\langle\rho v_{w}>\right.$ represents the cost of providing flow control. The time average and the Root Mean Square of $\left\langle\rho v_{w}\right\rangle$ are determined from a flow history of several cavity characteristic times $T D / U_{\infty}$. The time mean component $\overline{\left\langle\rho v_{w}\right\rangle}$ corresponds to the usage of compressor bleed air or other high pressure air supply in an aircraft store bay in which a flow conditioning device based on air jets is implemented. This parameter is regarded in this study as a more significant indicator of the cost of flow control with respect the Root Mean Square component $<\rho v_{w}>_{R M S}$.

The cavity pressure drag coefficient $C_{d}$ is defined as

$$
C_{d}=\int_{-D}^{0} \frac{p(L, y)-p(0, y)}{\frac{1}{2} \rho_{\infty} U_{\infty}^{2} D} d y
$$

where $L$ is the cavity streamwise length, which is defined in Fig. 1. This definition is consistent with previous work* The time mean drag coefficient $\overline{C_{d}}$ reflects the surface averaged steady streamwise loads on the cavity structure, while the Root Mean Square component $C_{d R M S}$ gives an indication of the unsteady aerodynamic streamwise loading. $C_{d R M S}$ is therefore a good measurement of the steadying effects of air injection on the flow.

At the beginning of the baseline flow time dependent computation, a streamwise uniform boundary layer is imposed above the cavity opening and uniform stagnation flow conditions are set inside the enclosure. A small vortical disturbance is added at the beginning of the computation at the base of the boundary layer to trigger the cavity flow instability. ${ }^{27}$ Upon reaching the cavity rear bulkhead, this convected vortex is sheared, perturbing the flow inside the enclosure and generating new disturbances in the neighbourhood of the

\footnotetext{
*In Rona and Brooksbank, ${ }^{14}$ a $D$ was omitted in typesetting the denominator of Eq. 19.
}

$\bar{K}$
\begin{tabular}{|c|ccc|}
\hline 2.55 & Cav0.25-2.55 & & \\
2.00 & Cav0.25-2.00 & Cav0.5-2.00 & Cav0.75-2.00 \\
1.72 & & & Cav0.75-1.72 \\
\hline \multirow{2}{*}{} & 0.25 & 0.50 & 0.75 \\
\cline { 2 - 4 } & & $d / D$ & \\
\end{tabular}

Table 1 Summary of test cases.

upstream bulkhead. These amplify in the shear flow along the top opening and develop into a self-sustained instability without any additional periodic nor impulsive numerical forcing. This way, the time-dependent model self-selects the dominant instability mode. At the selected flow conditions, this is the second cavity mode. ${ }^{23}$ This mode has a measured frequency ${ }^{23}$ $f=599 \mathrm{~Hz}$ and a Strouhal number $S t r=f U_{\infty} / D$ of 0.312 . The computation is time marched over several non-dimensional characteristic times $T D / U_{\infty}$, where $1 T D / U_{\infty}=1 / S t r=3.20$, until a statistically stationary flow regime is obtained.

The numerical tests to attenuate the cavity flow oscillations by injection are started from the statistically stationary self-sustained flow regime of the baseline flow. This approach evaluates the effectiveness of the suppression strategy in damping a fully developed flow instability. After starting the injection at $0 \leq d \leq 0.75 D$, the numerical prediction is time marched by about $5 T D / U_{\infty}$ and then the effects of flow control are evaluated.

\section{RESULTS}

\section{Cavity drag and flow control}

Six different air injection configurations have been modelled, including the baseline case with zero injection mass flow rate. In this study, the designation Cav $\hat{d}-\bar{K}$ identifies each test case by its normalised upstream perforated wall depth $\hat{d}=d / D$ and by the applied time mean back wall injection pressure ratio $\bar{K}=p_{0} / p_{\infty}$. Table 1 specifies the $(\hat{d}, \bar{K})$ matrix of the five cavity models where injection was used.

Table 2 presents a summary of the drag coefficients and injection mass flow rates determined from the five flow models with injection and from the baseline cavity model. Cav0.25-2.00, Cav0.5-2.00 and Cav0.75-2.00 show the effects of changing the slot depth $d$ while maintaining the same injection pressure $p_{0}=2 p_{\infty}$. Injecting air via the shallowest slot $d=0.25 D$ reduces the time mean drag coefficient $\overline{C_{d}}$ by about one third with respect to the baseline cavity $\overline{C_{d}}$. Widening the slot progressively reduces $\overline{C_{d}}$ down to $11 \%$ of the baseline value at $d=0.75 D$. The $d=0.75 D$ configuration predicted the lowest value of both $\overline{C_{d}}$ and $C_{d R M S}$ among the six tests and points towards a deep slot being the preferred configuration to achieve low steady and unsteady aerodynamic loads in the en-

3 of 10

American Institute of Aeronautics and Astronautics 


\begin{tabular}{|l|c|c|c|}
\hline & $\overline{C_{d}}$ & $C_{d R M S}$ & $\overline{\left\langle\rho v_{w}>\right.}$ \\
\hline Baseline & 0.4221 & 0.807 & 0 \\
\hline Cav0.25-2.00 & 0.2926 & 1.400 & 0.0197 \\
\hline Cav0.5-2.00 & 0.1035 & 0.493 & 0.0251 \\
\hline Cav0.75-2.00 & 0.0507 & 0.273 & 0.0369 \\
\hline Cav0.25-2.55 & 0.3921 & 1.340 & 0.0251 \\
\hline Cav0.75-1.72 & 0.7798 & 0.327 & 0.0254 \\
\hline
\end{tabular}

Table 2 Comparison of drag and mass flow rate coefficients for a Mach 1.5 cavity flow with and without mass injection.

closure. The cost of conditioning the cavity flow in Cav0.25-2.00, Cav0.5-2.00 and Cav0.75-2.00 is linked to the time mean injection mass flow rate $\left\langle\rho v_{w}\right\rangle$. This is shown in Table 2 to increase almost proportionally with slot depth $d$.

The two folds variation in mean mass injection flow rate among the three test cases at $p_{0}=2 p_{\infty}$ has significant implications towards a practical application of an air jet control device, for instance, in an aircraft store bay. Selecting an appropriate slot depth is likely to depend mainly on the level or pressure fluctuations or $C_{d R M S}$ that are deemed acceptable in the bay. Still, such injection has to be affordable in terms of the air mass flow rate supplied to the control jet. It is therefore of interest to compare air jet configurations where approximately the same amount of $\left\langle\rho v_{w}\right\rangle$ is used, as this enables to compare suppression performances at about equal cost of flow control. In Cav0.25-2.55 and Cav0.75-1.72, the injection back pressure has been adjusted to obtain about the same $\overline{\left\langle\rho v_{w}\right\rangle}$ as in Cav0.5-2.00, as indicated in Table 2. The predictions from these three tests indicate that a monotonic reduction in $C_{d R M S}$ is achieved by increasing the slot depth $d$. However, the time mean drag coefficient associated to the test with the deepest slot $d=0.75 D$ is worst than the one from the baseline flow prediction.

While the results in Table 2 may be deemed sufficient to guide the initial selection of a cavity flow control configuration, it is of interest to analyse in more detail time dependent flow predictions from the six test cases, to identify how flow injection attenuates the cavity instability and how this attenuation mechanism may be enhanced. This is discussed in the remainder of the paper.

\section{Baseline flow}

Figure 3 shows four density contour snapshots from time-dependent baseline flow predictions. The time sequence of Figure 3(a-d) documents large amplitude instabilities being convected in the downstream direction along the cavity opening and on the downstream bulkhead. These convected instabilities originate from the separating boundary layer at the upstream cavity edge. They develop in the shear layer that spans
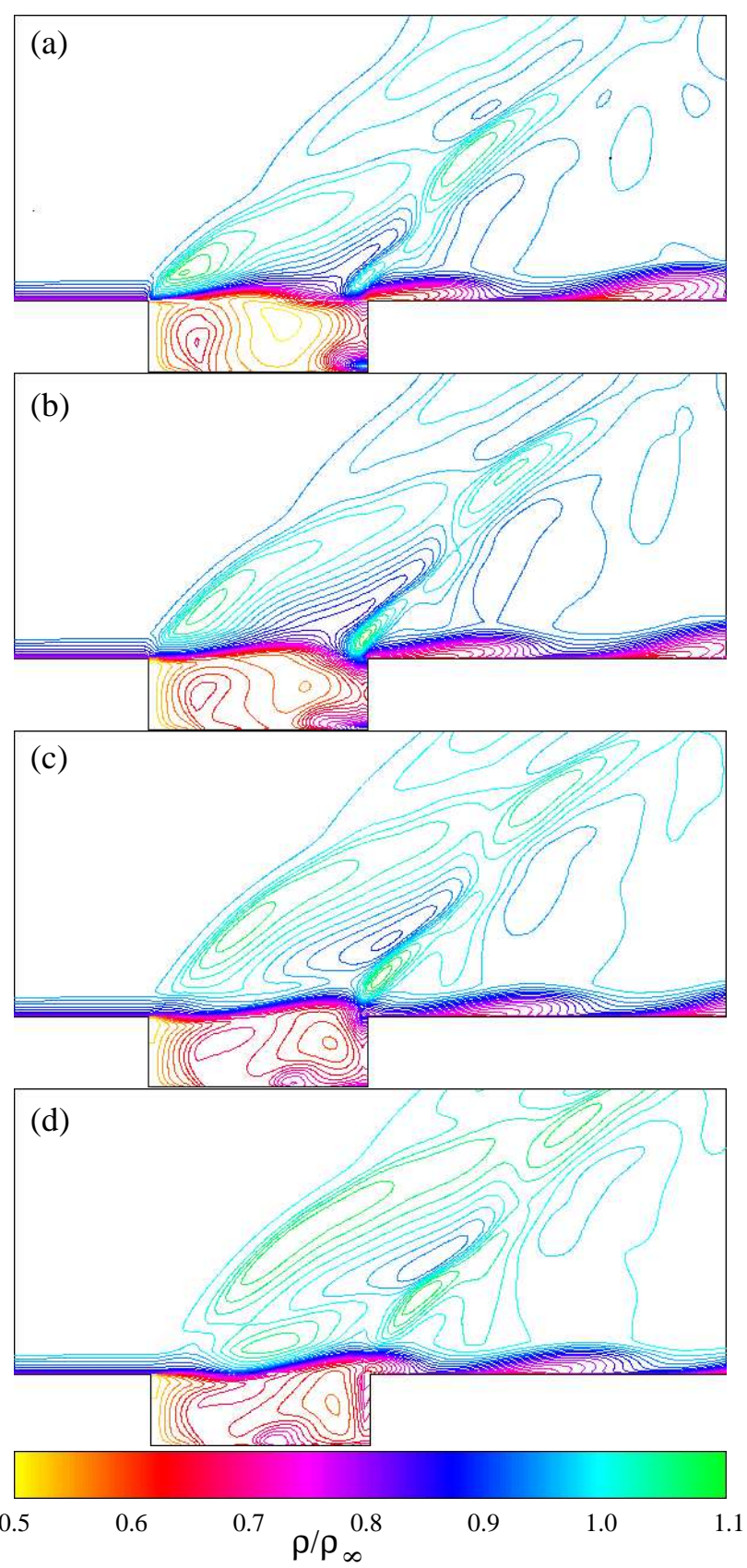

Fig. 3 Baseline flow density contour snapshots over one typical instability cycle.

across the enclosure in this 'open' cavity flow. Figure 3(a) captures one of such convected shear layer instabilities centred at about mid-span across the enclosure, while two other large scale flow structures are convected in the reattached boundary layer above the downstream bulkhead.

The turbulent boundary layer approaching the enclosure remains relatively undisturbed by the unsteady shear layer fluctuations downstream of it, supporting the current interpretation of the shear layer unsteadiness being essentially a convected Kelvin-Helmholtz type instability. This unsteadiness therefore requires

4 of 10

American Institute of Aeronautics and Astronautics 
a feed-back mechanism to become self-sustained.

In the time advancing sequence of Fig. 3(a-d), the shear layer fluctuation at the cavity mid-span in Fig. 3(a) advances towards the trailing edge in Fig. 3(b) and in Fig. 3(c), moving over it in Fig. 3(d). A small flow-normal shear layer fluctuation, close to the leading edge, identifies in Fig. 3(d) the onset of the next shear layer instability cycle. The presence of this upstream wave together with the downstream wave that has not yet fully cleared the downstream edge indicates that a mode wave number of two is captured in the time sequence of Fig. 3(a-d). This prediction agrees with the experimental observations by Zhang ${ }^{23}$ of the second cavity mode being dominant over the base mode ${ }^{23}$ in this test case.

As the shear layer oscillates in and out of the enclosure along the top open boundary, it interacts with the trailing edge geometry, generating alternating phases of mass injection and mass ejection. Figure 3(b) captures the mass injection phase, during which the high speed flow above the enclosure bends inwards, towards the cavity floor, adding mass and momentum to the enclosed flow. Figure 3(d) captures the mass ejection phase, during which flow exits from the enclosure, past the training edge. The flow normal position of the shear layer is an important parameter in determining the rate of the mass flow injection and ejection at the trailing edge. This is highlighted in the flow predictions with mass injection presented later on.

The impingement of the high speed flow against the downstream edge during mass injection causes an upstream travelling pressure wave inside the enclosure. The momentum inflow also drives a clockwise recirculation in the enclosure. Vorticity and pressure waves resulting from this flow-trailing edge interaction perturb the shear layer at the leading edge, providing a feed-back mechanism to self-sustain the instability.

\section{Flow control at constant injection pressure}

As injection is applied through the top $25 \%$ of the upstream bulkhead, the streamwise momentum in the separating boundary layer is enhanced. Figures 4(a) and $4(\mathrm{~b})$ indicate that, with this injection configuration, the cavity flow responds essentially like the baseline case, exhibiting a large-scale shear layer longitudinal instability characterised by a dominant second mode. The mass addition through the perforated upstream bulkhead over-fills the cavity and displaces the upstream portion of the top shear layer towards the free stream, strengthening the leading edge shock. This is documented by the greater packing of density contours above the upstream edge in Fig. 4(a) compared to Fig. 3(b).

The streamwise momentum added by mass injection reinforces the shear layer impingement on the trailing edge, strengthening the trailing edge shock in Fig. 4(a)
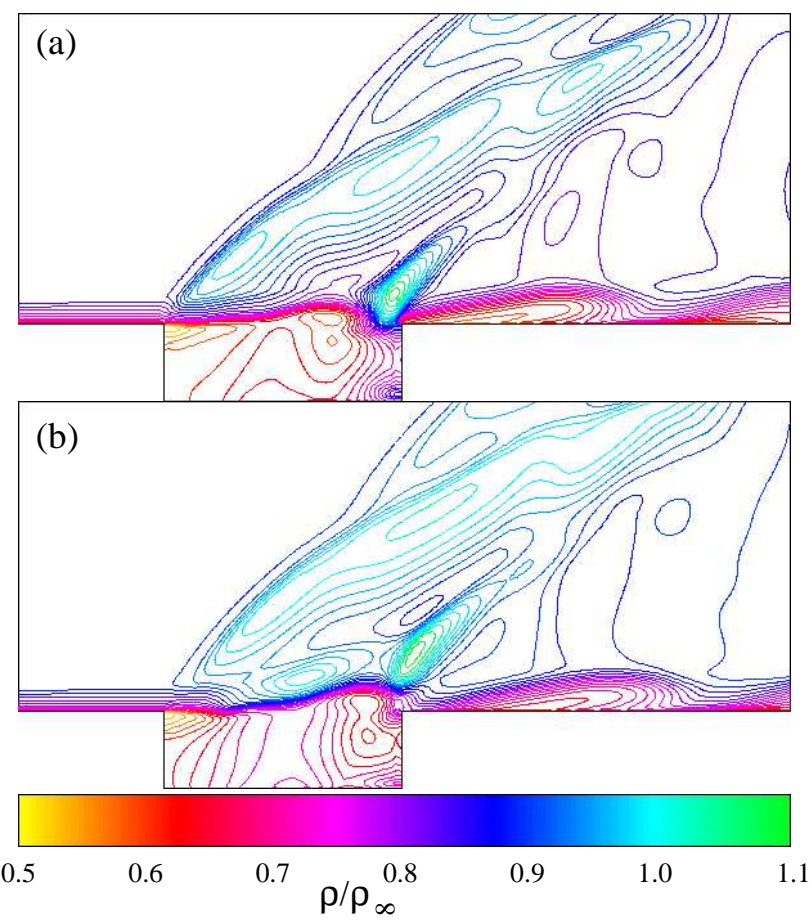

Fig. 4 Cav0.25-2.00 density contour snapshots over one typical instability cycle.

with respect to the baseline prediction in Fig. 3(b). This is likely to enhance wall pressure fluctuations on the cavity trailing edge wall, explaining the observed increase in $C_{d R M S}$ for Cav0.25-2.00 with respect to the baseline case, as documented in Table 2.

Increasing the injection depth to $d=0.50$ while maintaining the same injection back wall pressure $p_{0}=$ $2.00 p_{\infty}$ results in additional and substantial changes to the baseline flow pattern, compared to the shallower $d=0.25 D$ injection configuration. Specifically, the volume of air added through the upstream bulkhead displaces the downstream portion of the cavity shear layer outwards, in the flow normal direction, as shown by the density snapshots of Fig. 5(a-d). Most of the high-speed flow across the opening overflows the trailing edge, substantially reducing its interaction with the enclosure geometry. This is particularly noticeable during the mass injection phase of the cavity flow instability cycle, in Fig. 5(d). The weakening of the flow-trailing edge interaction tends to remove the feed-back point in the baseline cavity instability mechanism. Specifically, in the baseline cavity, the frequency of the main instability mode is dictated by a phase match between a downstream convecting shear layer Kelvin-Helmholtz type instability and an upstream travelling pressure wave inside the enclosure. The forward path of the instability loop features a Kelvin-Helmholtz wave leaving the neighbourhood of the upstream edge to impinge against the trailing edge. The feed-back path includes a pressure wave leaving the trailing edge to perturb the shear layer at the lead-

5 of 10

American Institute of Aeronautics and Astronautics 

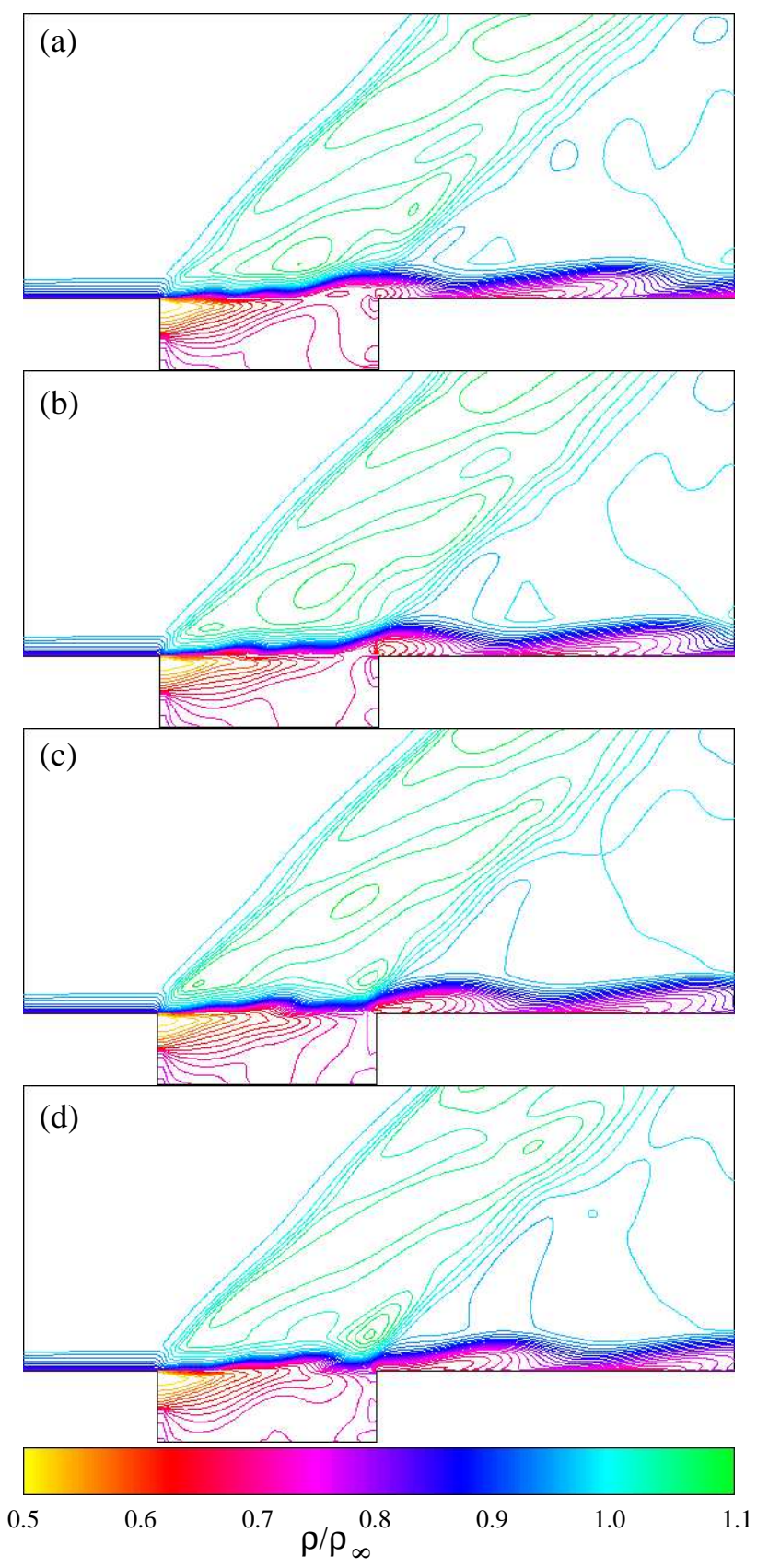

Fig. 5 Cav0.5-2.00 density contour snapshots over one typical instability cycle.

ing edge. The length of the enclosure provides a fixed reference point to promote phase-coherent oscillations, retaining modal energy, like in an edge tone flow instability. Suppressing the trailing edge interaction point introduces dispersion in the dominant instability mode feed-back mechanism, leaking modal energy away from the baseline main mode that weakens. Some evidence of this dissipation mechanism is given in the context of the results for Cav0.75-2.00, presented later on.

During mass ejection, Fig. 5(a-b), a volume of fluid comparable to the one in Fig. 3(d) leaves the enclosure, as the volume of the control flow injected through the upstream bulkhead compensates for some attenuation in the baseline shear layer flow-normal fluctuations around the trailing edge during this phase of the instability cycle. The leading edge shock is more welldefined in the time sequence of Fig. 5(a-d), compared to the corresponding feature in Fig. 3(a-d). This is in part due to the attenuation of the shear layer periodic flow-normal displacement beneath the shock and by the lifting of the shear layer time mean position away from the enclosure. This creates a ramp effect at the cavity leading edge that reinforces the leading edge shock.

Further increasing the injection depth to $d=$ $0.75 D$ enhances the flow control mechanism displayed by Cav0.5-2.00. The density contour snapshots of Fig. 6(a-d) document an enhanced lifting of the shear layer time mean position above the opening. The shear layer displays an attenuated self-sustained instability at the second cavity mode and the sequence of Fig. 6(a-d) captures four phases of this mode, which are equispaced in time. In the neighbourhood of the downstream edge, the shear flow stays clear from the cavity wall in all four phases of this mode. The reduced flow impingement at the trailing edge weakens the pressure feed-back mechanism in the enclosure and leads to modal energy dispersion. Some evidence of this dispersion is shown by the presence of short ripples in the shear layer in Fig. 6(a-d), indicating higher wavenumber modes co-existing with a suppressed second mode in the time-dependent flow predictions.

\section{Flow control at constant injection mass flow rate}

Figures 5, 7 and 8 show the variation in the predicted time dependent cavity density field as the injection area is increased from $d=0.25 D$ to $d=0.75 D$ while the injection mass flow rate is maintained approximately constant at $\left\langle\rho v_{w}\right\rangle \simeq 0.152$. This maintains the same control mass flux among the three test cases but progressively reduces the streamwise control momentum flux at increasing $d / D$. The time sequence of Fig. 7(a-d) clearly shows a well-developed cavity second mode, similar to Fig. 4(a-b). Increasing the injection area progressively attenuates this instability. With the $d=0.50 D$ injection slot, most of the unsteady wave activity in the high speed flow above the enclosure is reduced. Specifically, in Fig. 7, a forward moving pressure wave is shown running along the leading edge shock to the far field, as indicated by the arrow. This wave is angled at about $\arcsin \left(2.5^{-1}\right)$ to the free stream and is part of the far-field acoustic radiation from the enclosure, which is an important by-product of the cavity fluid dynamic unsteadiness. Figure 8 shows the suppressed flow instability that is obtained using a $0.75 D$ deep injection slot and a steady back pressure of $p_{0}=1.72 p_{\infty}$. These density contour snapshots correspond to the time sequence of 

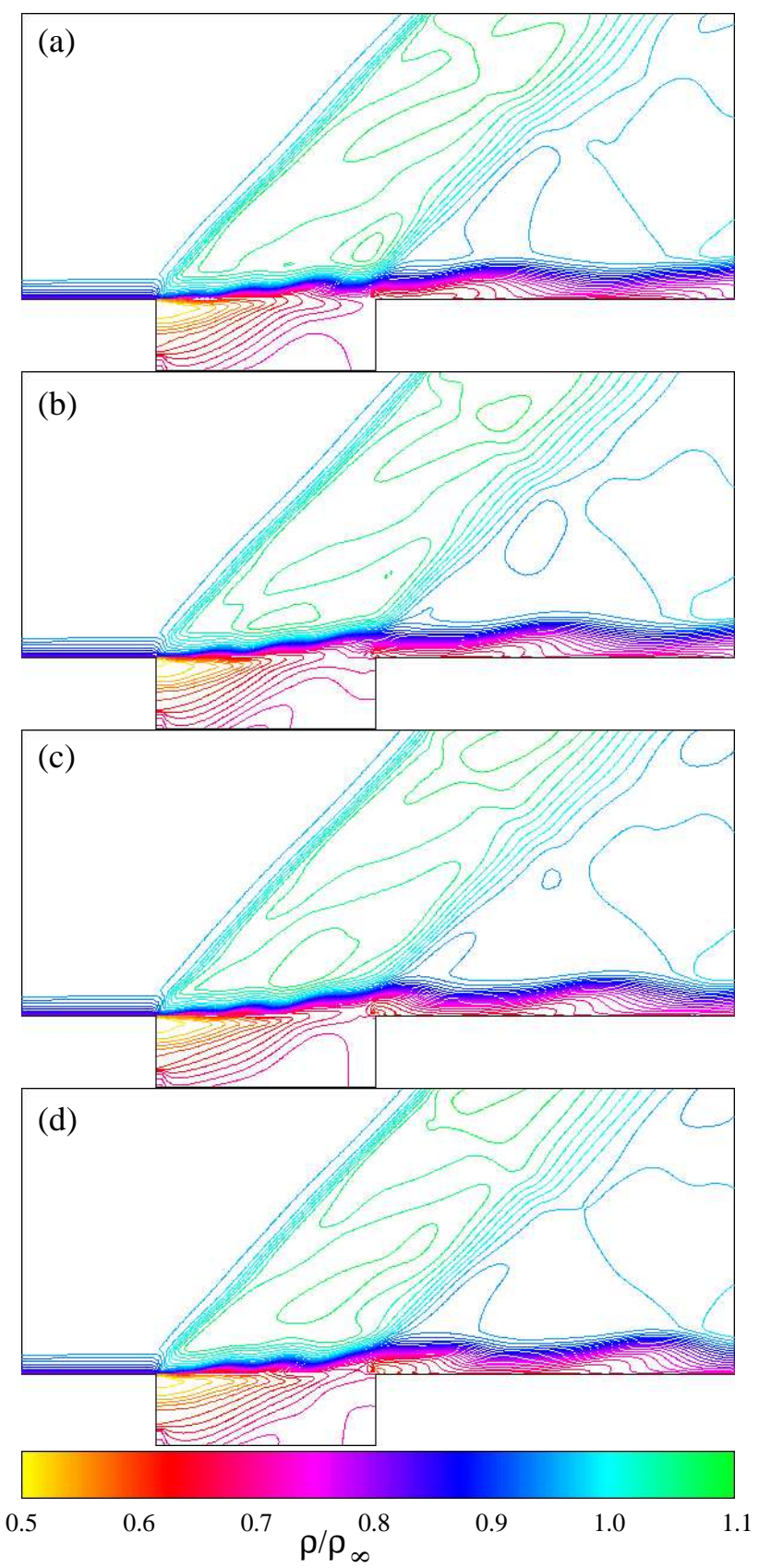

Fig. 6 Cav0.75-2.00 density contour snapshots over one typical instability cycle.

Figure 3(a-d) and highlight a reduced shear layer oscillation above the cavity opening and over the downstream bulkhead. Figure 8(a-d) shows that the shear layer has been lifted in the flow-normal direction by the added mass from the model air jet. In the shear layer, the convected structures display a more modest interaction with the downstream cavity edge than in the baseline flow. The injection has also suppressed most of the large-scale flow recirculation in the enclosure and the unsteadiness of the wave system in the supersonic flow above it. Lifting the shear layer mean position has created a ramp effect on the flow
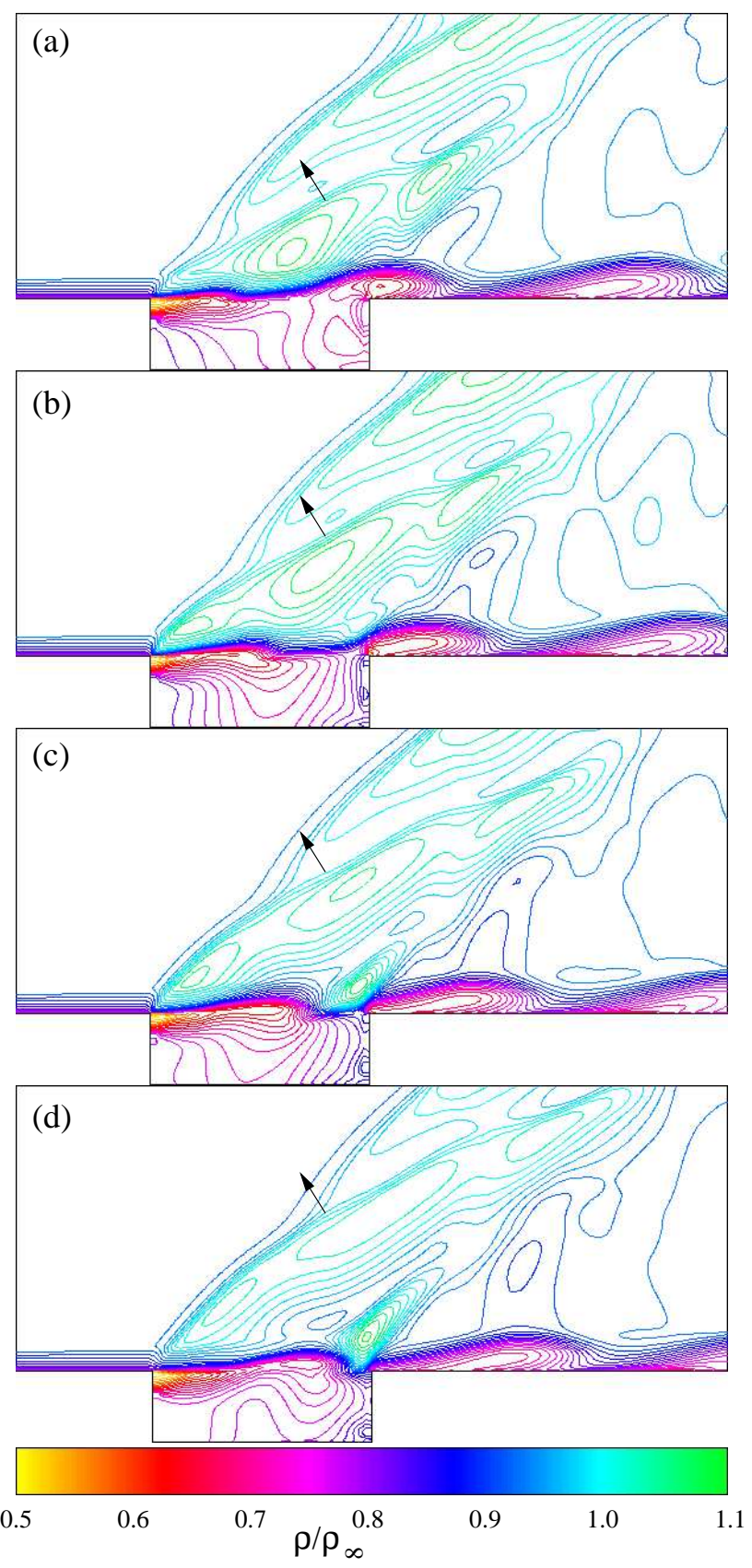

Fig. 7 Cav0.25-2.55 density contour snapshots over one typical instability cycle.

approaching the enclosure at the cavity leading edge. This has reinforced the leading edge shock, as shown by the greater density contour packing at this location. However, this reinforcement in Cav0.75-1.72 is milder than in Cav0.75-2.00. This is a desirable feature, as a weaker cavity leading edge shock results in a lower contribution to airframe wave drag.

Further details of the changes in the time-dependent cavity flow due to the deepening of the injection slot can be observed from the predicted velocity fields in the enclosure. A selection of velocity vector plots from the three test cases with the same time mean injection 

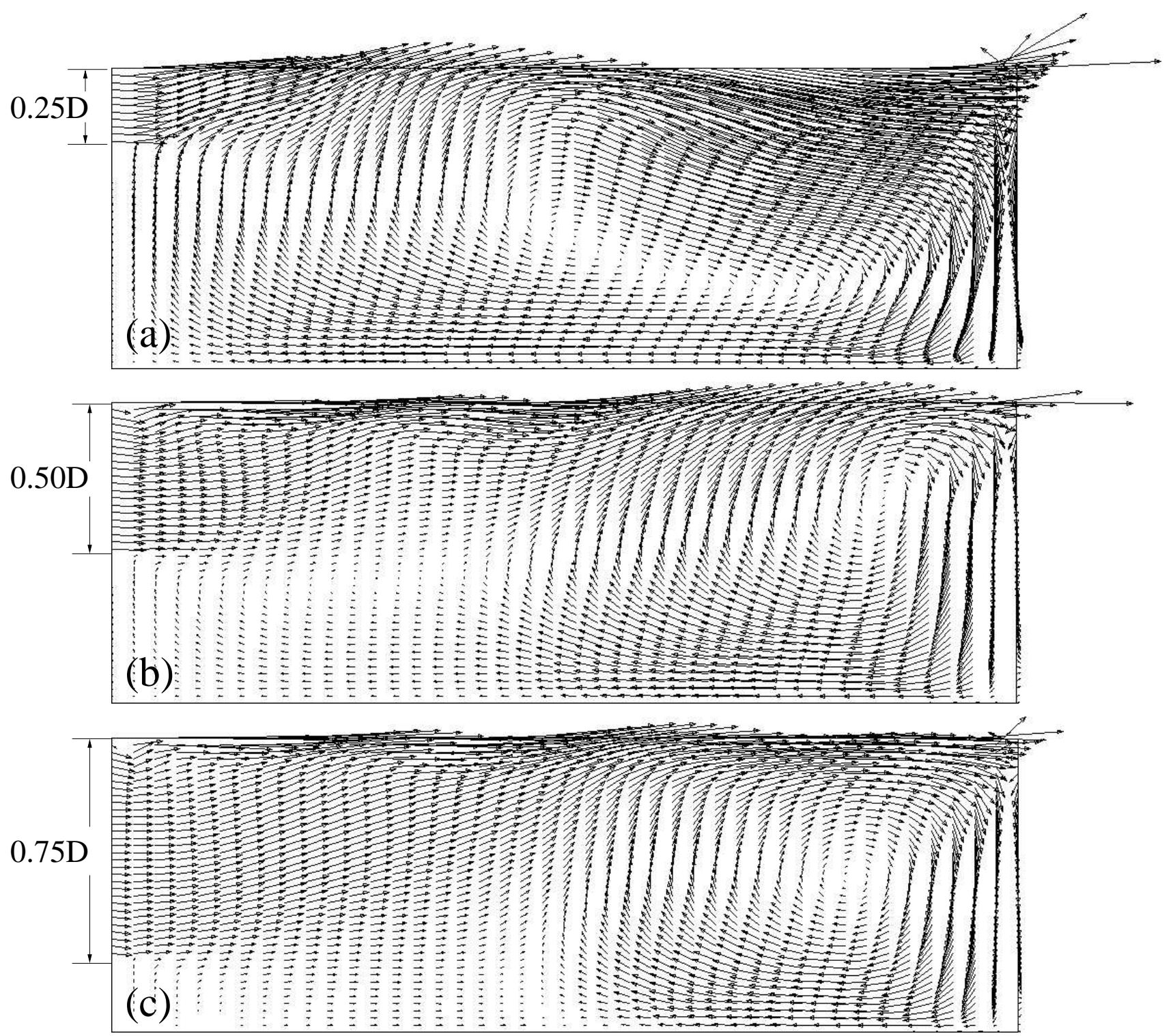

Fig. 9 Velocity vector snapshots inside the enclosure at the same mean injection mass flow rate: (a) $d=0.25 D$, (b) $d=0.50 D$, (c) $d=0.75 D$.

mass flow rate is reported in Fig. 9(a-c). These predictions highlight significant differences in flow dynamics within the cavity among Cav0.25-2.55, Cav0.5-2.00 and Cav0.75-1.72. As air is injected through the shallowest perforated wall, Fig. 9(a), the air momentum increases the flow-normal velocity gradient in the shear layer, adding to the creation and convection of vorticity by Kelvin-Helmholtz type instabilities. Confining the injection to a small area at the top of the upstream bulkhead also promotes the baseline large-scale clockwise recirculation in the enclosure. This recirculation features an upstream moving air stream along the cavity floor. This air stream extends to the full length of the cavity and occupies almost the whole bottom quarter of the enclosure, in the range $-0.75 D \geq y \geq-1 D$.

As the injection area is increased to $d=0.5 D$,
Fig. 9(b), a lower flow-normal velocity gradient is set in the shear layer, which is accompanied by an increased shear layer thickness. The thicker shear layer is more stable. Specifically, it would be expected for the maximum amplification growth rate of Kelvin-Helmholtz type instabilities to occur at lower wavenumbers, possibly below the baseline cavity most amplified instability mode. This leads to the observed reduction of the unsteadiness in the flow and this occurrence is confirmed by the reduction of flow-normal velocity peaks along the cavity opening. Under this flow injection configuration, a large-scale recirculation persists inside the enclosure which is more confined to the downstream half of the cavity. Increasing the injection depth to $d=0.75 D$ further reduces the flow-normal velocity gradient and creates effectively two shear flow 


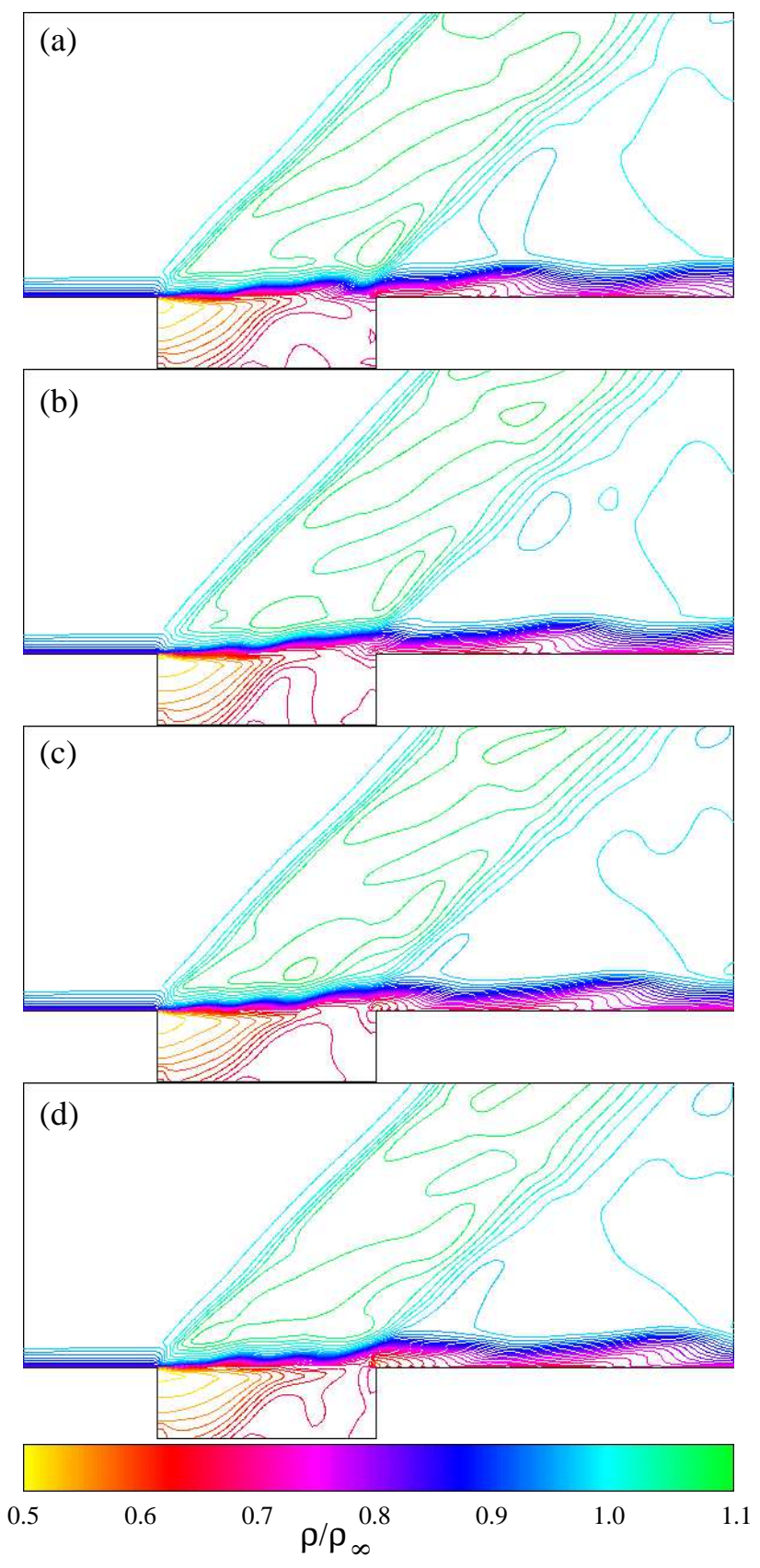

Fig. 8 Cav0.75-1.72 density contour snapshots over one typical instability cycle.

regions, one along the top of the cavity and one along $y=-0.75 D$, as shown by Fig. $9(\mathrm{c})$. In between these two regions of shear, the flow is essentially moving downstream with very small vorticity, rising towards the top of the enclosure to lift the top shear layer mean line. The clockwise flow recirculation in the cavity has also been reduced. Specifically, this injection configuration has modified the upstream moving air stream close to the cavity floor. In Fig. 9(a), this upstream recirculation runs over the full length of the cavity floor. In Fig. 9(c), the $d=0.75 D$ slot injection has suppressed this recirculation in the range $0 D \leq x \leq 0.5 L$ and has confined it to the downstream half of the enclosure.

\section{CONCLUSIONS}

Time-dependent modelling of a supersonic turbulent cavity flow was performed to investigate different cavity flow instability suppression layouts involving downstream mass injection from the upstream bulkhead. Specifically, the effects of varying the injection slot depth and the injection back pressure were considered. The cost of implementing this flow control technique was included in the analysis by monitoring the predicted time mean mass flow rate through the control air jets. ${ }^{14}$

Time dependent flow predictions show that some configurations are more effective than others in reducing the dominant mode of the self-sustained large-scale flow instability that naturally occurs at the selected inflow conditions. By increasing the injection slot depth and back pressure, reductions of the unsteady loads in the enclosure were predicted, as indicated by $C_{d R M S}$, that were accompanied by variations of the cavity mean drag coefficient, sometimes of opposite sign.

Density and velocity vector predictions give some insight into the instability suppression mechanism triggered by the air jets. Mass injection stabilises the flow by modifying both the vorticity and the feed-back pressure fluctuations in the enclosure, which are key components in the baseline cavity self-sustained instability.

The main changes to the pressure field arise from the lifting of the shear layer across the cavity opening. This enables the shear layer to overflow the trailing edge, rather than periodically impinge against it. This tends to remove the downstream feed-back point in the cycle of the self-sustained cavity flow instability, which is attenuated. The reduced pressure fluctuations at the trailing edge attenuate the noise radiated to the far field, which is a welcome by-product of this cavity instability suppression strategy.

The changes to the cavity vorticity field due to mass injection play an equally important role in reducing the flow unsteadiness. Mass injection affects the shear layer vorticity thickness. In the injection configuration with the deepest slot, $d=0.75 D$, the velocity gradient in the shear layer is reduced and this should shift the peak growth rate of the shear layer KelvinHelmholtz instability with respect to the the cavity dominant second Rossiter mode. Inside the enclosure, increasing the injection depth $d$ significantly affects the upstream flow recirculation along the cavity floor, which, in the $d=0.75 D$ test, becomes confined to the downstream half of the cavity. This results in a lower $C_{d R M S}$ prediction and indicates that, at the selected test conditions, a deeper injection area is more effec-

9 of 10

American Institute of Aeronautics and Astronautics 
tive in attenuating the leading mode instability than a shallower jet with the same time mean injected mass flow rate.

\section{ACKNOWLEDGEMENTS}

The support of EPSRC grant GR/N23745 is acknowledged. The author acknowledges Mr. Steven Foster for producing the numerical results.

\section{REFERENCES}

${ }^{1}$ Stanek, M. J., Raman, G., Kibens, V., Ross, J. A., Odedra, D., and Peto, J. W., "Control of cavity resonance through very high frequency forcing," Paper 2000-1905, AIAA, Jan. 2000.

${ }^{2}$ Arunajatesan, S., Shipman, J. D., and Sinha, N., "Hybrid RANS-LES simulation of cavity flow fields with control," Paper 2002-1130, AIAA, Jan. 2002.

${ }^{3}$ Montegau, L., Kook, H., and Franchek, M. A., "Active control of flow-induced cavity resonance," Paper 98-2349, AIAA/CEAS, Jun. 1998.

${ }^{4}$ Lamp, A. M. and Chokani, N., "Control of cavity resonance using steady and oscillatory blowing," Paper 99-0999, AIAA, Jan. 1999.

${ }^{5}$ Williams, D. R., Rowley, C., Colonius, T., Murray, R., MacMartin, D., Fabris, D., and Albertson, J., "Model-based control of cavity oscillations - Part 1: Experiments," Paper 2002-0971, AIAA, Jan. 2002.

${ }^{6}$ Grace, S. M., "An overview of computational aeroacoustic techniques applied to cavity noise prediction," Paper 2001-0510, AIAA, Jan. 2001.

${ }^{7}$ Shaw, L. L., "Weapons bay acoustic environment control," Paper 95-141, CEAS/AIAA, Jun. 1995.

${ }^{8}$ Rona, A., Aerodynamic and aeroacoustic estimations of oscillatory supersonic flows, Ph.D. thesis, University of Southampton, UK, Jun. 1997.

${ }^{9}$ Rona, A., Chen, X. X., Zhang, X., and Edwards, J. A., "Control of cavity flow oscillations through leading edge flow modifications," Paper 98-0672, AIAA, Jan. 1998.

${ }^{10}$ Zhang, X., Rona, A., and Edwards, J. A., "The effect of trailing edge geometry on cavity flow oscillation driven by a supersonic shear layer," The Aeronautical Journal, Vol. 102, No. 1013, May 1998, pp. 129-136.

${ }^{11}$ Zhang, X., Chen, X. X., Rona, A., and Edwards, J. A., "Attenuation of cavity flow oscillation through leading edge flow control," Journal of Sound and Vibration, Vol. 221, No. 1, 1999, pp. $23-47$.

${ }^{12}$ Smith, B. R., Welterlen, T. D., Maines, B. H., Shaw, L. L., Stanek, M. J., and Grove, J. E., "Weapons bay acoustic suppression from rod spoilers," Paper 2002-0662, AIAA, Jan. 2002.

${ }^{13}$ Grove, J. E., Birkbeck, R. M., and Kreher, J. M., "Acoustic and separation characteristics with bay leading edge blowing," Paper 2000-1904, AIAA/CEAS, Jun. 2000.

${ }^{14}$ Rona, A. and Brooksbank, E. J., "Injection parameters for an effective passive control of cavity flow instability," Paper 2002-0119, AIAA, Jan. 2002.

${ }^{15}$ Stanek, M. J., Raman, G., Kibens, V., Cain, A., and Lepicovsky, J., "Advanced actuator concepts for active aeroacoustic control," Paper 2000-1930, AIAA, Jan. 2000.

${ }^{16}$ Fabris, D. and Williams, D. R., "Experimental measurements of cavity and shear layer response to unsteady bleed forcing," Paper 99-0605, AIAA, Jan. 1999.

${ }^{17}$ Rowley, C. W., Colonius, T., and Murray, R. M., "POD based models of self-sustained oscillations in the flow past an open cavity," Paper 2000-1969, AIAA, Jan. 2000.

${ }^{18}$ Rowley, C. W., Williams, D. R., Colonius, T., Murray, R. M., MacMartin, D. G., and Fabris, D., "Model-based control of cavity oscillations Part II: System identification and analysis," Paper 2002-0972, AIAA, Jan. 2002.

${ }^{19}$ Brooksbank, E. J. and Rona, A., "POD analysis and prediction of cavity flow instability," ICSV9 Paper 700, Ninth International Congress on Sound and Vibration, Orlando, Florida, USA, Jul. 2002.

${ }^{20}$ Rona, A. and Brooksbank, E. J., "POD analysis of cavity flow instability," Paper 2003-0178, AIAA, Jan. 2003.

${ }^{21}$ Charwat, A. F., Roos, J. N., Dewey, F. C. J., and Hitz, J, A., "An investigation of separated flow - Part I: The pressure field," J. of the Aerospace Sciences, Vol. 28, Jun. 1961, pp. 457470.

${ }^{22}$ Zhang, X. and Edwards, J. A., "An investigation of supersonic oscillatory cavity flows driven by thick shear layers," The Aeronautical Journal, Vol. 94, No. 940, Dec. 1990, pp. 355-364.

${ }^{23}$ Zhang, X., "Compressible cavity flow oscillation due to shear layer instabilities and pressure feedback," AIAA Journal, Vol. 33, No. 8, Aug. 1995, pp. 1404-1411.

${ }^{24}$ Zhang, X. and Edwards, J. A., "Analysis of unsteady cavity flow employing an adaptive meshing algorithm," Computers 8 Fluids, Vol. 25, No. 4, May 1996, pp. 373-393.

${ }^{25}$ Zhang, X., An experimental and computational investigation into shear layer driven single and multiple cavity flowfields, Ph.D. thesis, University of Cambridge, UK, 1988.

${ }^{26}$ Zhang, X., Rona, A., and Edwards, J. A., "An observation of pressure waves around a shallow cavity," J. Sound and Vibration, Vol. 214, No. 4, Jul. 1998, pp. 771-778.

${ }^{27}$ Rona, A. and Dieudonné, W., "Unsteady laminar and turbulent cavity flow models by second order upwind methods," Paper 99-0656, AIAA, Jan. 1999.

${ }^{28}$ Roe, P. L., "Approximate Riemann solvers, parameter vectors, and difference schemes," J. Comp. Physics, Vol. 43, 1981, pp. 357-372.

${ }^{29}$ Roe, P. L., "Some contributions to the modeling of discontinuous flows," Lectures in Applied Math. 22, Am. Math. Soc., 1985.

${ }^{30} \mathrm{Kok}$, J. C., "Resolving the dependence on free-stream values for the k-omega turbulence model," AIAA Journal, Vol. 38, No. 7, Jul. 2000, pp. 1292-1295.

${ }^{31}$ Rona, A. and Bennett, W. P., "Non-uniform total temperature in a turbulent vortex street," Paper 2001-2467, AIAA, Jun. 2001.

${ }^{32}$ Jeng, Y. N. and Payne, U. J., "Numerical study of a supersonic open cavity flow and pressure oscillation control," Journal of Aircraft, Vol. 32, No. 2, March 1995, pp. 363-369. 